Table 1 HSPA5 haplotype frequencies in bipolar disorder

\begin{tabular}{|c|c|c|c|c|c|c|c|c|}
\hline \multirow[b]{3}{*}{ Haplotype } & \multicolumn{5}{|c|}{ Japanese case-control samples } & \multicolumn{3}{|c|}{ NIMH trios $(n=88)$} \\
\hline & \multirow{2}{*}{$\begin{array}{l}\text { Controls } \\
(n=254)\end{array}$} & \multicolumn{2}{|c|}{ All cases $(n=195)$} & \multicolumn{2}{|c|}{ With family history $(n=67)$} & \multirow[b]{2}{*}{ Freq. $\mathrm{T}$} & \multirow[b]{2}{*}{ Freq. NT } & \multirow[b]{2}{*}{$P$} \\
\hline & & Cases & $P$ & Cases & $P$ & & & \\
\hline 1: C-del-C & 0.116 & 0.135 & 0.375 & 0.141 & 0.426 & 0.074 & 0.069 & 0.835 \\
\hline 2: C-del-T & 0.076 & 0.128 & 0.010 & 0.201 & 0.000084 & 0.0057 & 0 & 0.239 \\
\hline 3: C-G-C & 0.368 & 0.325 & 0.185 & 0.276 & 0.043 & 0.38 & 0.41 & 0.583 \\
\hline 4: T-del-T & 0.439 & 0.410 & 0.388 & 0.380 & 0.222 & 0.52 & 0.51 & 0.914 \\
\hline
\end{tabular}

Other haplotypes estimated from the NIMH samples were less than $2 \%$. Haplotypes consist of three polymorphisms $(-307 \mathrm{C} \rightarrow$ T-rs3216733-rs 12009$)$. Freq. T or Freq. NT indicates the frequency of the transmitted or nontransmitted haplotype, respectively. $P$ values were calculated by COCAPHASE.

transcription start site), rs3216733

$(\mathrm{Gdel})$, and rs12009 $(\mathrm{C} \rightarrow \mathrm{A}))$ in Japanese case-control samples (described previously ${ }^{1}$ ), we found that haplotype 2 (C-del-T) was significantly associated with bipolar disorder $(P=0.010)$. This association was stronger in affected individuals with family history $(P=0.000084$; Table 1$)$. This risk haplotype was extremely rare in the NIMH trio samples, and no association was found (Table 1).

These findings suggest that genetically determined interindividual variability of ER stress-response does relate to bipolar disorder, that there may be functional polymorphisms in other ER stress-response-related genes, in addition to the -116 polymorphism of $X B P 1$, and that genetic risk factors may differ among populations.

The antimalaria drug mefloquine, which often causes an episode of depression or mania in susceptible individuals, was recently reported to cause ER stress in the brain $^{2}$. Further investigations of the ER stress-response signaling system in the pathophysiology of bipolar disorder is warranted.

\section{ACKNOWLEDGMENTS}

Data and biomaterials of the NIMH pedigrees were collected in four projects that participated in the NIMH Bipolar Disorder Genetics Initiative. From 1991-1998, the Principal Investigators andCoInvestigators were as follows: J. Nurnberger, M. Miller and E. Bowman (Indiana University); T. Reich, A. Goate and J. Rice (Washington University); J.R. DePaulo Jr., S. Simpson and C. Stine (Johns Hopkins University); and E. Gershon,
D. Kazuba and E. Maxwell (NIMH Intramural Research Program).

Chihiro Kakiuchi ${ }^{1}$, Shinichiro Nanko ${ }^{2}$, Hiroshi Kunugi $^{3}$ \& Tadafumi Kato ${ }^{1}$

${ }^{1}$ Laboratory for Molecular Dynamics of Mental Disorders, Brain Science Institute, RIKEN, Wako, Saitama 351-0198, Japan. ${ }^{2}$ Department of Psychiatry, Teikyo University School of Medicine, Itabashi, Tokyo 173-8605, Japan. ${ }^{3}$ Department of Mental Disorder Research, National Institute of Neuroscience, Kodaira, Tokyo 187-8502, Japan. Correspondence should be addressed to T.K. (kato@brain.riken.jp).

1. Kakiuchi, C. et al. Impaired feedback regulation of XBP1 as a genetic risk factor for bipolar disorder. Nat. Genet. 35, 171-175 (2003).

2. Dow, G.S., Hudson, T.H., Vahey, M. \& Koenig, M.L. The acute neurotoxicity of mefloquine may be mediated through a disruption of calcium homeostasis and ER function in vitro. Malar. J. 2, 14 (2003).

\title{
A national DNA bank in The Gambia, West Africa, and genomic research in developing countries
}

To the editor:

The Gambian National DNA Bank, the first National Bio-Bank developed in Africa, was funded in November 2000 by the Medical Research Council (MRC) as one of 14 DNA collection sites established to study the genetics of complex diseases. One of these sites is housed at the MRC Laboratories in The Gambia and has a special, though not exclusive, focus on malaria, HIV and tuberculosis. Additional projects include analyses of genome diversity in West African populations and a collection of twin-sister pairs to study the genetic basis of dizygotic twins ( $\sim 2 \%$ of live births in the country). So far, more than 30,000 DNA samples have been collected, with many ongoing studies and more planned.
For the first time in a sub-Saharan country, a centralized structure and database for archiving DNA samples has been created, in collaboration with the Jean Dausset Foundation-CEPH. The bank is regulated by guidelines (Supplementary Note online) for sample collection, archiving, data storage and privacy protection, which were developed and approved by the MRC, the MRC Laboratories Scientific Coordinating Committee and by the Gambia Government/MRC Joint Ethics Committee. The Guidelines, which are enforced by these Committees, stemmed from the need to adapt to the local reality the many existing recommendations on bio-banking, privacy protection, genetics research and, generally, on medical research in developing countries (http://www3.who.int/whosis/ genomics/pdf/genomics08.pdf, http://www.mrc.ac.uk/pdf-devsoc.pdf, http://www.mrc.ac.uk/pdf-tissue_guide_fin. pdf, http://www.nuffieldbioethics.org/ publications/pp_0000000013.asp).

The Gambian DNA Bank promotes sharing of information and resources with centers around the world, and one of its ultimate goals is health improvement. In the short term, benefits should accrue to the participants in the studies. A recent example is a large project on genetic and environmental factors for susceptibility to tuberculosis, designed as a household association and family-based study and carried out in The Gambia, Guinea-Bissau and Guinea-Conakry ${ }^{1}$. The project focused on the systematic detection of tuberculosis cases in the families of individuals with tuberculosis and controls. Clinical services 
were considerably augmented within the framework of the genetic study, to the benefit of the populations involved.

During research projects at the MRC Unit in The Gambia, biological samples (mostly blood, buffy coats and peripheral blood mononuclear cells) were taken and stored at a time when molecular genetics was still in its infancy. The process of obtaining consent has evolved enormously over time, and in the past, samples may have been taken from subjects who were not made specifically aware that the samples were going to be used for genetic studies. The Guidelines now regulate the use of these archived DNA specimens: in almost all circumstances they can be used only after anonymization and unlinking (Supplementary Note online), and all use requires approval by the Ethics Committee.

Regrettably, most African countries do not have the infrastructure necessary to carry out genomic studies, and so most scientific exchange has taken place with developed countries. The Ethics Committee has a key role in sanctioning shipment of any samples to external collaborators. The Committee evaluates the specificity of the project and reevaluates projects in case their focus changes over time. All transfer of DNA samples to collaborators is accompanied by signed agreements and detailed documentation describing the projects involved. The review of sample transfer to third parties is compulsory and is not permitted from one collaborator to another without consent of the Ethics Committee.
In the long term, understanding genetic determinants of infection can lead to prevention and improved treatment, but this does not mean that genetic research will cure infectious diseases. Even more complicated is the situation of population-genetics studies promoted by interest in the greater genomic diversity found in Africans compared with Europeans and Asians, and consistent with the hypothesis of an African origin of modern humans. Where sampling is not part of research aimed directly at disease, the connection to health interventions seems weak. But this does not imply that studies on genome diversity should not be done, for the contribution of both inter- and intraethnic genetic variability comparisons is crucial in our understanding of immunity to infections. For example, the findings that West African Fulani have fewer malaria attacks and lower parasitemia by Plasmodium falciparum than two sympatric ethnic groups ${ }^{2}$, and that they have intragroup variation in immune responses to malaria ${ }^{3}$, open the possibility of identifying genetic factors determining malarial immunity. Immunogenetic associations with infectious disease, such as the association between HLA-B53 and resistance to severe malaria found in Gambian children ${ }^{4}$, can affect vaccine development.

The ethics guidelines adopted for the Gambian National DNA Bank do not presume to be the last word on the matter. Rather, we hope their publication will stimulate discussion of the appropriate way to regulate genomic research in developing countries. We hope that our initiative will be seen as an important experience in a complex and fast-evolving venture from which Africa should not be excluded, and as a possible template to be adapted by similar initiatives in other parts of the world.

Note: Supplementary information is available on the Nature Genetics website.

\section{ACKNOWLEDGMENTS}

This work was supported by the MRC (UK) to G.S.

Giorgio Sirugo ${ }^{1}$, Maarten Schim Van Der Loeff ${ }^{1}$, Omar Sam², Ousman Nyan ${ }^{3}$, Margaret Pinder ${ }^{1}$, Adrian V Hill, Dominic Kwiatkowski ${ }^{4}$, Andrew Prentice ${ }^{1,5}$, Claudia de Toma ${ }^{6}$, Howard M. Cann ${ }^{6}$, Mathurin Diatta ${ }^{1}$, Muminatou Jallow ${ }^{1,7}$, Gareth Morgan ${ }^{1}$, Malcolm Clarke ${ }^{8}$, Tumani Corrah ${ }^{1}$, Hilton Whittle ${ }^{1}$ \& Keith McAdam ${ }^{1,5}$

${ }^{1}$ MRC Laboratories, PO Box 273, Banjul, The Gambia. ${ }^{2}$ Department of State for Health, Banjul, The Gambia. ${ }^{3}$ University of The Gambia, School of Medicine, The Gambia. ${ }^{4}$ Wellcome Trust Centre for Human Genetics, University of Oxford, UK.

${ }^{5}$ London School of Hygiene and Tropical

Medicine, UK. ${ }^{6}$ Fondation Jean-Dausset-CEPH, Paris, France. ${ }^{7}$ Royal Victoria Teaching Hospital, Banjul, The Gambia. ${ }^{8}$ Gambian Government / MRC Laboratories Joint Ethics Committee, Fajara, The Gambia. Correspondence should be addressed to G.S. (gsirugo@mrc.gm).

1. Lienhardt, C. et al. Am. J. Epidemiol. 155 1066-1073 (2002).

2. Modiano, D. et al. Proc. Natl. Acad. Sci. USA 93, 13206-13211 (1996).

3. Luoni, G. et al. Genes Immun. 2, 411-414 (2001).

4. Hill, A.V. et al. Nature 352, 595-600 (1991).

\section{Evidence for lateral gene transfer from salmonids to two Schistosome species}

\author{
To the editor: \\ Two detailed studies of the genomes of Asian \\ and African and American schistosomes were \\ recently published ${ }^{1,2}$. In the former, some \\ 13,131 gene clusters from Schistosoma \\ japonicum EST clones were analyzed and \\ their hypothetical protein products were \\ compared with those of model organisms, \\ uncovering about $30 \%$ homologous proteins \\ (at $\mathrm{E} \leq 10^{-10}$ ). In their report on the \\ evolutionary implications of their \\ sequencing project ${ }^{1}$, however, the authors \\ did not mention that one of their libraries \\ (SjC7/94) contained many inserts with high \\ homology to genomic DNA of salmonid fish. \\ This was recorded in GenBank, in which it \\ was stated that these homologous inserts
}

amounted to $2-3 \%$ of the library clones. Our analysis showed that more than $10 \%$ of the SjC7/94 clones matched $\left(\mathrm{E} \leq 10^{-10}\right)$ DNA of salmonid fish; more than $95 \%$ of these were the best matches found in the entire nr database.

Our analysis of the SjC7/94 library also showed that nearly all of the salmonidhomologous inserts are found in noncoding regions of the salmonid genes and seem to comprise salmon-specific mobile genetic elements. For example, the clone BU712912 contains a 480-bp sequence that is almost identical ( $94 \%$ identity; $\mathrm{E}=0$ ) to the Oncorhynchus mykiss LINE Rsg-1 (ref. 3); searches of the mammalian database for similar sequences produced no matches
$(E>0.05)$, and none were found in other invertebrates $(\mathrm{E}>0.06)$. The SjC7/94 library also contains sequences homologous to the salmonid SINEs SmaI (found only in Oncorhynchus keta and Oncorhynchus gorbuscha, possibly after lateral transfer ${ }^{4}$; $\mathrm{E}=90^{-50}$ ) and $\mathrm{HpaI}$ (found in all salmonids; $\left.\mathrm{E}=10^{-78}\right)$. There are even $S$. japonicum sequences homologous $\left(\mathrm{E}=10^{-27}\right)$ to FokI, which is supposed to be specific to the genus Salvelinus ${ }^{4,5}$; these show a higher level of identity than is seen in other salmonids.

To verify the presence of these sequences in the schistosome genome and to elucidate more about their expression, we carried out PCR using primers designed to amplify six of these salmonid-specific sequences, initially 